\title{
Quantification of Dopamine Transporter in Human Brain Using PET with ${ }^{18}$ F-FE-PE2I
}

\author{
Takeshi Sasaki1 ${ }^{1,2}$, Hiroshi Ito ${ }^{1,3}$, Yasuyuki Kimura ${ }^{1}$, Ryosuke Arakawa $^{1}$, Harumasa Takano $^{1}$, Chie Seki $^{3}$, \\ Fumitoshi Kodaka ${ }^{1}$, Saori Fujie ${ }^{1}$, Keisuke Takahata ${ }^{1}$, Tsuyoshi Nogami ${ }^{1}$, Masayuki Suzuki ${ }^{1}$, Hironobu Fujiwara ${ }^{1}$, \\ Hidehiko Takahashi ${ }^{1}$, Ryuji Nakao ${ }^{4}$, Toshimitsu Fukumura ${ }^{5}$, Andrea Varrone ${ }^{4}$, Christer Halldin ${ }^{4}$, Toru Nishikawa ${ }^{2}$, \\ and Tetsuya Suhara ${ }^{1}$

\begin{abstract}
${ }^{I}$ Molecular Neuroimaging Program, Molecular Imaging Center, National Institute of Radiological Sciences, Chiba, Japan; ${ }^{2}$ Department of Psychiatry and Behavioral Sciences, Tokyo Medical and Dental University Graduate School, Tokyo, Japan; ${ }^{3}$ Biophysics Program, Molecular Imaging Center, National Institute of Radiological Sciences, Chiba, Japan; ${ }^{4}$ Karolinska Institutet, Department of Clinical Neuroscience, Centre for Psychiatry Research, Karolinska Hospital, Stockholm, Sweden; and ${ }^{5}$ Molecular Probe Program, Molecular Imaging Center, National Institute of Radiological Sciences, Chiba, Japan
\end{abstract}

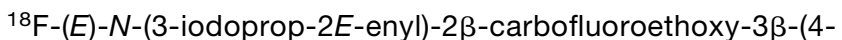
methylphenyl)nortropane ( $\left.{ }^{18} \mathrm{~F}-\mathrm{FE}-\mathrm{PE} 2 \mathrm{I}\right)$ is a new $\mathrm{PET}$ radioligand with a high affinity and selectivity for the dopamine transporter (DAT). In nonhuman primates, ${ }^{18} \mathrm{~F}-\mathrm{FE}-\mathrm{PE} 2 \mathrm{I}$ showed faster kinetics and less production of radiometabolites that could potentially permeate the blood-brain barrier than did ${ }^{11} \mathrm{C}-\mathrm{PE} 2 \mathrm{I}$. The aims of this study were to examine the quantification of DAT using ${ }^{18} \mathrm{~F}-\mathrm{FE}-\mathrm{PE} 2 \mathrm{l}$ and to assess the effect of radiometabolites of ${ }^{18} \mathrm{~F}-\mathrm{FE}-\mathrm{PE} 2 \mathrm{l}$ on the quantification in healthy humans. Methods: A 90-min dynamic PET scan was obtained for 10 healthy men after intravenous injection of ${ }^{18} \mathrm{~F}-\mathrm{FE}-\mathrm{PE} 2 \mathrm{I}$. Kinetic compartment model analysis with a metabolite-corrected arterial input function was performed. The effect of radiometabolites on the quantification was evaluated by time-stability analyses. The simplified reference tissue model (SRTM) method with the cerebellum as a reference region was evaluated as a noninvasive method of quantification. Results: After the injection of ${ }^{18} \mathrm{~F}-\mathrm{FE}-\mathrm{PE} 2 \mathrm{I}$, the whole-brain radioactivity showed a high peak $(\sim 3-5$ standardized uptake value) and fast washout. The radioactive uptake of ${ }^{18} \mathrm{~F}-\mathrm{FE}-\mathrm{PE} 2 \mathrm{I}$ in the brain was according to the relative density of the DAT (striatum $>$ midbrain $>$ thalamus). The cerebellum showed the lowest uptake. Tissue time-activity curves were well described by the 2-tissue-compartment model (TCM), as compared with the 1-TCM, for all subjects in all regions. Time stability analysis showed stable estimation of total distribution volume with 60 -min or longer scan durations, indicating the small effect of radiometabolites. Binding potentials in the striatum and midbrain were well estimated by the SRTM method, with modest intersubject variability. Although the SRTM method yielded a slight underestimation and overestimation in regions with high and low DAT densities, respectively, binding potentials by the SRTM method were well correlated to the estimates by the indirect kinetic method with $2-\mathrm{TCM}$. Conclusion: ${ }^{18} \mathrm{~F}-\mathrm{FE}-$
\end{abstract}

\footnotetext{
Received Dec. 6, 2011; revision accepted Feb. 27, 2012.

For correspondence or reprints contact: Hitoshi Ito, Biophysics Program, Molecular Imaging Center, National Institute of Radiological Sciences, 4-9-1, Anagawa, Inage-ku, Chiba, 263-8555, Japan.

E-mail: hito@nirs.go.jp

Published online Jun. 11, 2012.

COPYRIGHT @ 2012 by the Society of Nuclear Medicine, Inc.
}

PE2I is a promising PET radioligand for quantifying DAT. The binding potentials could be reliably estimated in both the striatum and midbrain using both the indirect kinetic and SRTM methods with a scan duration of $60 \mathrm{~min}$. Although radiometabolites of ${ }^{18} \mathrm{~F}-\mathrm{FE}-\mathrm{PE} 2 \mathrm{I}$ in plasma possibly introduced some effects on the radioactivity in the brain, the effects on estimated binding potential were likely to be small.

Key Words: ${ }^{18} \mathrm{~F}-\mathrm{FE}-\mathrm{PE} 2 \mathrm{I}$; positron emission tomography; dopamine transporter; kinetic modeling; radiometabolite

J Nucl Med 2012; 53:1065-1073

DOI: 10.2967/jnumed.111.101626

D opamine transporter (DAT) plays a crucial role in the regulation of dopamine concentration in the synaptic cleft by dopamine reuptake. Changes in the density and function of DAT have been reported in various neuropsychiatric disorders, such as Parkinson disease (1), Huntington disease (2), attention-deficit/hyperactivity disorder (3), autism (4), and schizophrenia (5). Although DAT ligands for SPECT have been widely used in clinical practice, developing a useful radioligand for PET — which has higher resolution and better ability of quantification than SPECT - is the key to assessing its role in the pathophysiology of these diseases and to developing new therapeutic approaches for them.

Several radioligands for imaging DAT have been developed and used for PET. Among ${ }^{11} \mathrm{C}$-labeled radioligands, ${ }^{11} \mathrm{C}$-cocaine (6), ${ }^{11} \mathrm{C}$-WIN35,428 (CFT) (7), ${ }^{11} \mathrm{C}-\beta-\mathrm{CIT}$ (8), and ${ }^{11} \mathrm{C}$-DL-threo-methylphenidate (9) have relatively low affinity for DAT or have slow kinetics in the highDAT-density regions. ${ }^{11} \mathrm{C}$-altropane has high affinity and selectivity for DAT (10), but the kinetics in the human brain have not been reported in detail to our knowledge. The ${ }^{18} \mathrm{~F}$-labeled radioligands that have been studied in humans so far include ${ }^{18} \mathrm{~F}-\mathrm{CFT}\left({ }^{18} \mathrm{~F}-\mathrm{WIN} 35,428\right)(11)$, $N$-3-fluoropropyl-2- $\beta$-carboxymethoxy-3- $\beta$-(4-iodophenyl) 


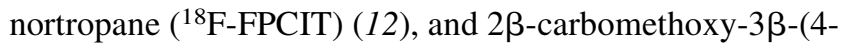
chlorophenyl)-8-(2-fluoroethyl)nortropane $\left({ }^{18} \mathrm{~F}-\mathrm{FECNT}\right)$ (13). All of them have high affinity and selectivity for DAT, but the kinetics are relatively slow, and more than 90 min are needed to reach peak uptake in the striatum.

Recently, a new ligand, $N$-(3-iodoprop-2E-enyl)-2 $\beta$ carbomethoxy-3 $\beta$-(4-methylphenyl)nortropane (PE2I), with a high affinity for DAT (inhibition constant, $17 \mathrm{nM}$ ) and good selectivity, was developed $(14,15)$. In human PET studies, ${ }^{11} \mathrm{C}$-PE2I showed a high specific-to-nonspecific ratio (15-18). However, 2 problems have been reported in quantifying DAT with ${ }^{11} \mathrm{C}$-PE2I. First, because of the relatively slow kinetics of ${ }^{11} \mathrm{C}-\mathrm{PE} 2 \mathrm{I}$ in the striatum, reference tissue methods severely $(\sim 50 \%)$ underestimated DAT binding in this region, compared with those by the methods with arterial input function $(16,17)$. Second, a radiometabolite of ${ }^{11} \mathrm{C}$ PE2I has been found to cross the blood-brain barrier (BBB) in rats, thus potentially reducing the accuracy of the quantification of DAT (19).

A fluoroethyl analog of PE2I, ${ }^{18} \mathrm{~F}-(E)-N$-(3-iodoprop- $2 E$ enyl)-2 $\beta$-carbofluoroethoxy-3 $\beta$-(4-methylphenyl)nortropane $\left({ }^{18} \mathrm{~F}-\mathrm{FE}-\mathrm{PE} 2 \mathrm{I}\right)$ (inhibition constant, $12 \mathrm{nM}$ ), has recently been developed and evaluated in nonhuman primates (20). In monkeys, ${ }^{18}$ F-FE-PE2I was more favorable for the quantitative analysis of DAT, because it showed faster kinetics and less production of BBB-permeable radiometabolites than did ${ }^{11} \mathrm{C}$-PE2I. The quantification of DAT with ${ }^{18} \mathrm{~F}$-FE-PE2I was less biased than that with ${ }^{11} \mathrm{C}$-PE2I (21).

The aims of this study were to examine the method for quantification of DAT using ${ }^{18} \mathrm{~F}-\mathrm{FE}-\mathrm{PE} 2 \mathrm{I}$ and to assess the effect of radiometabolites of ${ }^{18} \mathrm{~F}-\mathrm{FE}-\mathrm{PE} 2 \mathrm{I}$ on the quantification in healthy humans.

\section{MATERIALS AND METHODS}

\section{Subjects}

Ten healthy men (mean age $\pm \mathrm{SD}, 28.1 \pm 6.9 \mathrm{y}$; age range, 2039 y) participated in this study. All subjects were free of any somatic, neurologic, or psychiatric disorders. The study was approved by the Ethics and Radiation Safety Committee of the National Institute of Radiologic Sciences, Chiba, Japan. Written informed consent was obtained from all subjects before their inclusion in the study.

\section{PET Procedure}

${ }^{18}$ F-FE-PE2I was synthesized from its acid precursor through a reaction with ${ }^{18} \mathrm{~F}$-2-bromo-1-fluoroethane in dimethylformamide and sodium hydroxide in $N, N$-dimethylformamide, as previously described (22).

A 90-min dynamic scan was obtained for each subject after a 1-min intravenous injection of ${ }^{18}$ F-FE-PE2I using a PET scanner system (ECAT EXACT HR+; CTI-Siemens) The scan protocol consisted of 9 frames of $20 \mathrm{~s}, 5$ frames of $1 \mathrm{~min}, 4$ frames of $2 \mathrm{~min}, 12$ frames of $4 \mathrm{~min}$, and 5 frames of $6 \mathrm{~min}$. The injected dose and specific activity were $183.0 \pm 9.3 \mathrm{MBq}$ and $146.1 \pm$ 98.7 GBq/ $\mu \mathrm{mol}$ at the time of injection, respectively. A head holder was used to minimize head movements. Scatter correction was performed. Attenuation correction was based on a transmission scan using a ${ }^{68 / 68} \mathrm{Ge} / \mathrm{Ga}$ source.

\section{Arterial Blood Sampling and Metabolite Analysis}

Arterial blood samples were taken manually 32 times after the injection of radioligand to obtain an arterial input function. Each blood sample was centrifuged to obtain plasma and blood cell fractions, and the concentration of radioactivity in whole blood and plasma was measured.

The fractions of the parent and its radiometabolites in plasma were determined by high-performance liquid chromatography (HPLC) from 10 blood samples for each subject. Each plasma sample had acetonitrile added and then was centrifuged. The supernatant of the centrifuged sample was subjected to radio-HPLC analysis (column, $\mu$ Bondapak C18 [Waters]). Acetonitrile (90\%) (A) and phosphoric acid $(0.01 \mathrm{M})(\mathrm{B})$ were used as mobile phases, with a flow rate of $6.0 \mathrm{~mL} / \mathrm{min}$. Gradient elution was used with the following gradient profile: 0-4.5 min, 25/75-70/30 A/B; 4.5-8.0 min, 70/30-25/75 A/B; and 8.0-10.0 $\mathrm{min}, 25 / 75-25 / 75 \mathrm{~A} / \mathrm{B}$. Linear interpolation was used to calculate the fractions of the parent and radiometabolites for the blood samples without metabolite analysis.

\section{Image Analysis}

T1-weighted MR images acquired with a 1.5-T MRI scanner (Gyroscan NT; Philips) (1-mm-slice axial images; repetition time, $21 \mathrm{~ms}$; echo time, $9.2 \mathrm{~ms}$; and flip angle, $30^{\circ}$ ) were coregistered to the corresponding PET images. Manually drawn volumes of interest were based on the anatomic information of MR images. Then, these volumes of interest were applied to the dynamic PET images to extract time-activity curves for the putamen, caudate, ventral midbrain (including the substantia nigra and ventral tegmental area), thalamus, and cerebellum. All image and kinetic analyses were performed using PMOD (version 3.0; PMOD Technologies).

\section{Kinetic Analysis}

Standard 1- and 2-tissue-compartment models (TCMs) $(18,23)$ with an arterial input function (concentration of the parent in plasma) were used to estimate rate constants and total distribution volume $\left(V_{\mathrm{T}}\right)$ by an iterative nonlinear least-squares curve-fitting procedure without weighting. Linear interpolation was used to calculate the concentration of the parent in plasma at the time points of time-activity curves of tissue. The rate constants $K_{1}$ and $k_{2}$ represent the influx and efflux rates, respectively, for radioligand diffusion across the BBB. For 2-TCM, the rate constants $k_{3}$ and $k_{4}$ represent radioligand transfer between the compartments for nondisplaceable and specifically bound radioligand, respectively. $V_{\mathrm{T}}$ is equal to the ratio of the concentration of radioligand in tissue to that in plasma at equilibrium. Blood volume was fixed at $0.05 \mathrm{~mL} / \mathrm{mL}$ (24). The binding potential $\left(B P_{\mathrm{ND}}\right)$ of ${ }^{18} \mathrm{~F}-\mathrm{FE}-\mathrm{PE} 2 \mathrm{I}$ was quantified by the indirect kinetic method. We used the cerebellum as the reference brain region because of its negligible DAT density as shown in a human autoradiographic study (25) and an in vivo displacement study in monkeys $(20) . B P_{\mathrm{ND}}$ can be expressed as:

$$
B P_{\mathrm{ND}}=\left(V_{\mathrm{T}(\text { regions })} / V_{\mathrm{T}(\text { cerebellum })}\right)-1,
$$

where $V_{\mathrm{T}(\text { regions })}$ and $V_{\mathrm{T}(\text { cerebellum })}$ are $V_{\mathrm{T}}$ of target regions and the cerebellum, respectively.

\section{Time-Stability Analysis}

To investigate the effect of scan length on the estimation of $V_{\mathrm{T}}$, we analyzed PET data while truncating scan length. The scan length from 90 to $40 \mathrm{~min}$, with 10-min decrements, was analyzed to estimate $V_{\mathrm{T}}$ of varying scan lengths, using the parent concentration in plasma as the input function. For each region and duration, 
$V_{\mathrm{T}}$ was expressed as the percentage of the $V_{\mathrm{T}}$ value obtained with a 90-min scan length.

\section{Kinetic Analysis with Radiometabolite-Included Input Function}

To assess the effect of lipophilic radiometabolites, we tested an alternative input function consisting of the concentration of the parent and the lipophilic radiometabolite, 4-hydroxymethyl analog of the parent (M1), to estimate rate constants and $V_{\mathrm{T}}$.

\section{Simplified Reference Tissue Model (SRTM)}

${ }^{18}$ F-FE-PE2I binding was also quantified by the SRTM method with the cerebellum as a reference region. Assuming that both target and reference regions have the same level of nondisplaceable binding, and the kinetics in the target and reference regions can be described by $1-\mathrm{TCM}, B P_{\mathrm{ND}}$ is obtained by solving the convolution equation using a nonlinear least-squares fitting procedure (26). In this method, the parameters are reduced to $3: R_{1}$ (ratio of $K_{1}$ relative to the reference region), $k_{2}$, and $B P_{\mathrm{ND}}$. In addition, to investigate the applicability of shorter study durations, $B P_{\mathrm{ND}}$ values estimated by the indirect kinetic method and by the SRTM method with 60-min scanning data were compared with those estimated with 90-min scanning data.

\section{Statistical Analysis}

The goodness of curve fitting of models with different levels of complexity was compared using the Akaike information criterion (AIC) (27) and $F$ test. In a model with better fitting, AIC shows lower values. A $P$ value of less than 0.05 was considered significant for the $F$ test. The SE of kinetic parameters was given by the diagonal of the covariate matrix (28). Divided by the estimate of the parameter itself, SE was expressed as a percentage (SE/[estimates of the parameter]) and used to assess parameter identifiability. A smaller percentage indicates better identifiability. Pearson $r$ and linear regression analyses were used to assess correlations between $B P_{\mathrm{ND}}$ values estimated with the different approaches. A paired $t$ test was applied to assess the difference in $B P_{\mathrm{ND}}$ values between the indirect kinetic and SRTM methods.

\section{RESULTS}

\section{Brain Uptake}

After the injection of ${ }^{18} \mathrm{~F}-\mathrm{FE}-\mathrm{PE} 2 \mathrm{I}$, the radioactivity was distributed throughout the brain, with a high peak $(\sim 3-5$ SUV) and fast washout (Figs. 1 and 2A). The peak uptake occurred within $10 \mathrm{~min}$ in all regions. The rank order of radioactivity from approximately $15 \mathrm{~min}$ to the end of the scan was as follows: putamen and caudate $>>$ midbrain $>$ thalamus $>$ cerebellum. The uptake in the midbrain was visible as 2 distinct regions. Specific binding, which is the difference in radioactivity between target regions and the cerebellum, reached peak levels within approximately $30 \mathrm{~min}$ after injection in all target regions (Fig. 2B). The ratio of radioactivity in the striatum to that in the cerebellum reached a peak level $(\sim 7.0)$ approximately $60 \mathrm{~min}$ after the injection and remained at almost the same level thereafter (Fig. 2C).

\section{Plasma Analysis}

Reversed-phase HPLC analysis of plasma resulted in the separation of the parent and 2 major radiolabeled components (Fig. 3A). The peak with longest retention time corresponded to the parent, representing approximately $14 \%$ of plasma radioactivity at $30 \mathrm{~min}$ after injection (Fig. 3B). Of the 2 major radiolabeled components, one (M1) was retained longer, representing approximately $20 \%$ of plasma radioactivity at $30 \mathrm{~min}$ after injection. The M1-to-parent ratio was stable $(\sim 1.3-1.4)$ at 20 min after injection. Retention of the other one (M2) was shorter and consisted of 2 peaks, which were not sufficiently separated from each other, representing approximately $70 \%$ of the plasma radioactivity at $30 \mathrm{~min}$ after injection. The concentration of the parent showed a quick peak and fast washout in plasma (Fig. 3C).

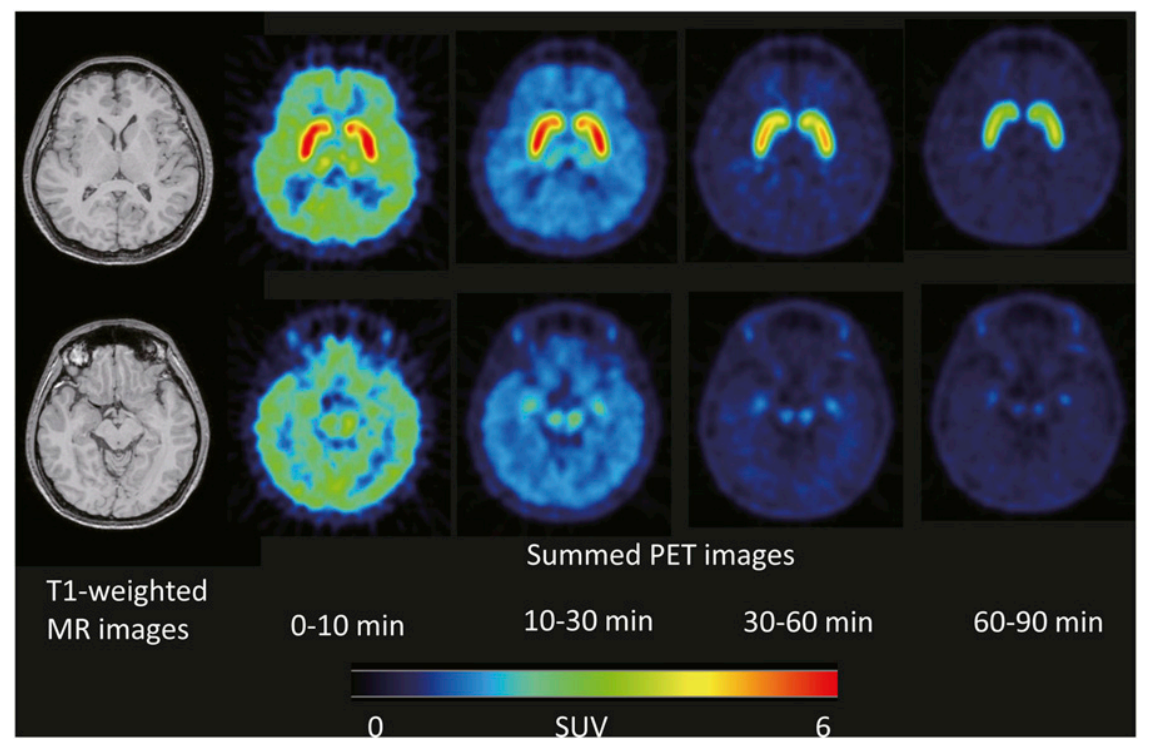

Quantification in Humans with ${ }^{18}$ F-FE-PE2I - Sasaki et al.
FIGURE 1. Representative dynamic PET images of healthy subject injected with ${ }^{18}$ F-FE-PE2I. PET images were created at level of striatum (top) and midbrain (bottom). SUV $=$ standardized uptake value. 

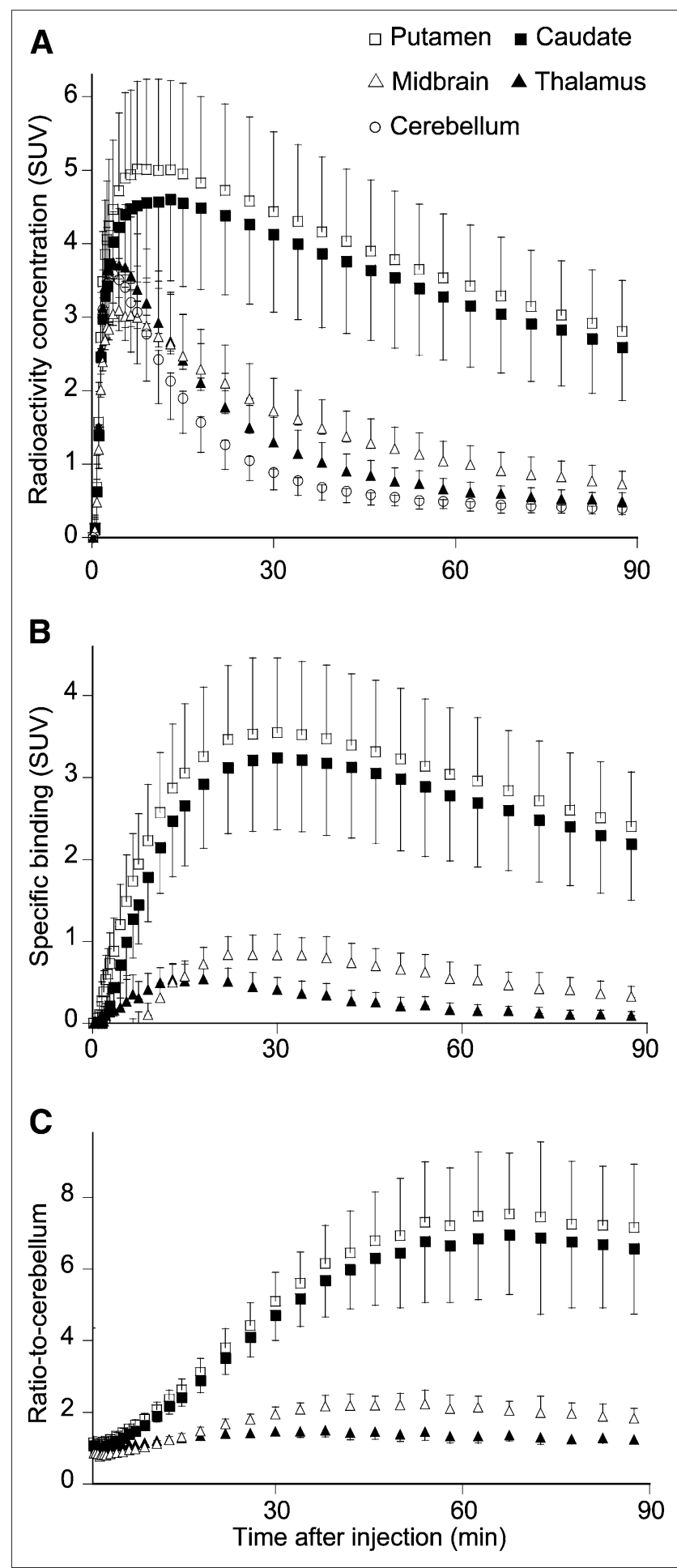

FIGURE 2. Average time course of radioactivity in brain regions after injection of ${ }^{18} \mathrm{~F}-\mathrm{FE}-\mathrm{PE} 2 \mathrm{I}$. Time course for regional radioactivity $(A)$, specific binding (B), and ratio to cerebellum (C). Data represent mean \pm SD of all 10 subjects. SUV $=$ standardized uptake value.

\section{Quantification of DAT by Compartment Analysis}

The 2-TCM provided significantly better fitting than the 1-TCM for all subjects in all regions. AIC of the 2-TCM was significantly lower than that of the 1-TCM in all

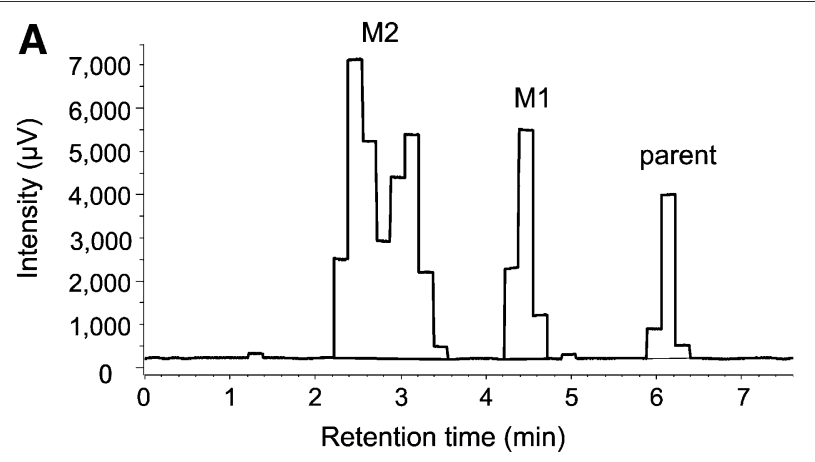

B

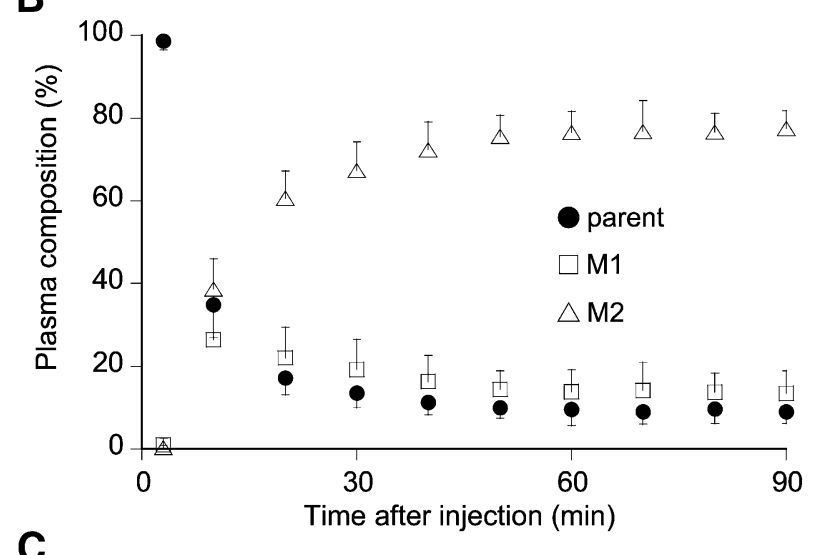

C

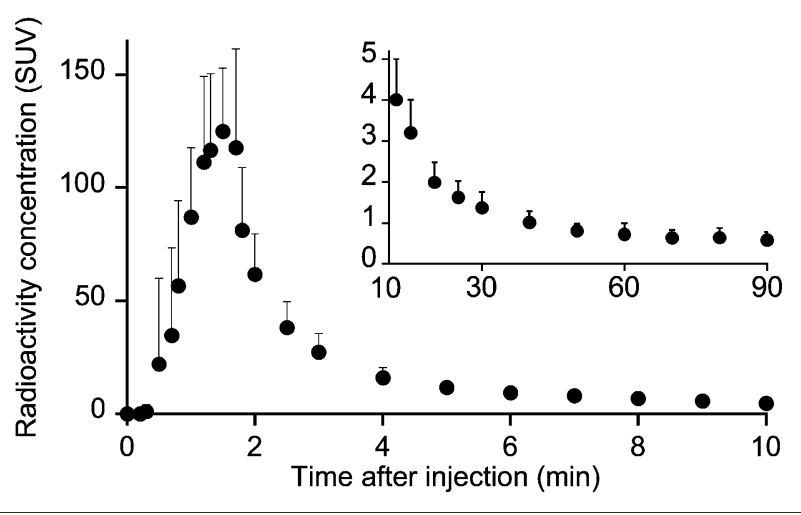

FIGURE 3. Concentration of ${ }^{18} \mathrm{~F}-\mathrm{FE}-\mathrm{PE} 2 \mathrm{l}$ and its composition in arterial plasma after injection of ${ }^{18} \mathrm{~F}-\mathrm{FE}-\mathrm{PE} 2 \mathrm{I}$. (A) Representative radiochromatogram at $30 \mathrm{~min}$ after injection of ${ }^{18} \mathrm{~F}-\mathrm{FE}-\mathrm{PE} 2 \mathrm{I}$. (B) Plasma composition of parent, M1, and M2. (C) Concentration of ${ }^{18} \mathrm{~F}-\mathrm{FE}-\mathrm{PE} 2 \mathrm{I}$ in plasma. Values from 0 to 10 and 10 to $90 \mathrm{~min}$ are shown in each graph with different ranges of $y$-axis. Data represent mean \pm SD of all 10 subjects. SUV $=$ standardized uptake value.

regions in all subjects (paired $t$ test, $P<0.05$ ). The $F$ test showed that the 2-TCM gave statistically better fittings than did the 1-TCM in all regions in all subjects $(F>$ $10.2, P<0.001)$. Thus, time-activity curves of all regions including the cerebellum were better described by the 2-TCM (Fig. 4).

The 2-TCM estimated $K_{1}$ and $V_{\mathrm{T}}$ with good identifiability $(1.8 \%$ and $3.7 \%$, respectively) (Table 1$) . B P_{\mathrm{ND}}$ values estimated by the indirect kinetic method were approximately $4.0-4.5$ in the putamen and caudate, approximately 0.5 in 


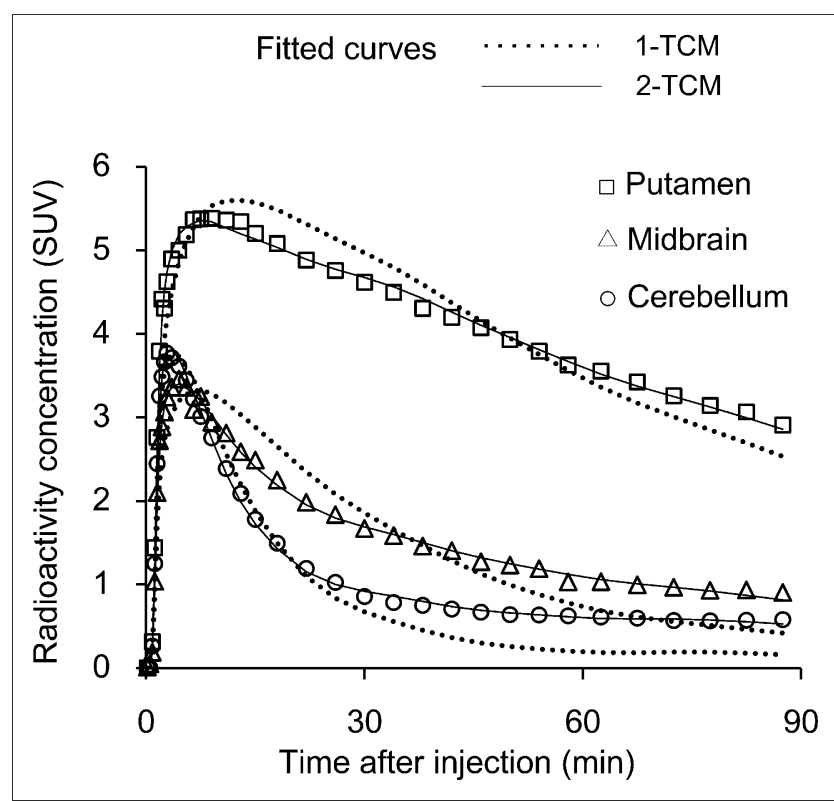

FIGURE 4. Representative fitted model curves of 1-TCM and 2-TCM. Time-activity curves in putamen, midbrain, and cerebellum were fitted to 1-TCM and 2-TCM using parent in plasma as input function. The 2-TCM (solid line) provided better fittings than 1-TCM (dotted line) for all 3 regions. SUV $=$ standardized uptake value.

the midbrain, and approximately 0.2 in the thalamus (Table 2). As to the intersubject variability, the coefficients of variation $(100 \times \mathrm{SD} /$ mean $)$ of the $B P_{\mathrm{ND}}$ values were approximately $20 \%-25 \%$ in the putamen and caudate, approximately $30 \%$ in the midbrain, and approximately $60 \%$ in the thalamus.

\section{Effects of Scan Length on Quantification of DAT}

$V_{\mathrm{T}}$ values were stably estimated using the 2-TCM with 60-min or longer scan length. In the putamen and cerebellum, $V_{\mathrm{T}}$ values gradually increased with longer scan length (Figs. 5A and 5B). With a 60-min scan length, $V_{\mathrm{T}}$ values were approximately $94 \%$ and $90 \%$ of those with full scan length in the putamen and cerebellum, respectively, indicating that $V_{\mathrm{T}}$ values were stably estimated with a $60-\mathrm{min}$ or longer scan length. Identifiability of $V_{\mathrm{T}}$ in the putamen improved over time, reaching approximately $2 \%$ at $90 \mathrm{~min}$, whereas in the cerebellum, it remained at almost the same level $(\sim 6 \%)$ at a 60 -min or longer scan length.

\section{Effects of Adding Lipophilic Radiometabolite to Input Function on Quantification of DAT}

To assess the effect of the lipophilic radiometabolite, we tested an alternative input function consisting of the concentration of the parent and M1, the lipophilic radiometabolite. Although the 2-TCM showed significantly better fitting than did the 1-TCM in the putamen, caudate, and midbrain based on AIC and the $F$ test, $V_{\mathrm{T}}$ was not well identified by the 2-TCM in the thalamus in some subjects and in the cerebellum in most subjects. In the putamen, caudate, and midbrain, $V_{\mathrm{T}}$ values estimated using the alternative input function were approximately 30\%-35\% lower than $V_{\mathrm{T}}$ values estimated with the parent concentration in plasma as the input function (Supplemental Table 1; supplemental materials are available online only at http:// jnm.snmjournals.org).

\section{Quantification of DAT with SRTM Method}

$B P_{\mathrm{ND}}$ values estimated by the SRTM method were approximately 3.6-4.0 in the putamen and caudate, approximately 0.6 in the midbrain, and approximately 0.3 in the thalamus (Table 2). Simple correlation analysis showed good correlation between $B P_{\mathrm{ND}}$ values estimated by the indirect kinetic method and by the SRTM method ( $r=$ $0.990, P<0.0001)$ (Fig. 6), although there was significant difference between these values $(P=0.0094$, paired $t$ test). A Bland-Altman plot showed that the SRTM method underestimated $B P_{\mathrm{ND}}$ in the high-density regions and overestimated $B P_{\mathrm{ND}}$ in the low-density regions; however, the magnitude of the bias was small $(\sim 10 \%$ in the putamen, caudate, and midbrain) (Supplemental Fig. 1). The intersubject variability of $B P_{\mathrm{ND}}$ by the SRTM method was

TABLE 1

Kinetic Parameters by 2-TCM Using Parent as Input Function

\begin{tabular}{|c|c|c|c|c|c|c|c|c|}
\hline Region & $\begin{array}{c}K_{1} \\
\left(\mathrm{~mL} \cdot \mathrm{cm}^{-3} \cdot \mathrm{min}^{-1}\right)\end{array}$ & $k_{2}\left(\min ^{-1}\right)$ & $k_{3}\left(\min ^{-1}\right)$ & $k_{4}\left(\min ^{-1}\right)$ & $\begin{array}{c}K_{1} / k_{2} \\
\left(\mathrm{~mL} \cdot \mathrm{cm}^{-3}\right)\end{array}$ & $k_{3} / k_{4}$ & $\begin{array}{c}V_{\mathrm{T}} \\
\left(\mathrm{mL} \cdot \mathrm{cm}^{-3}\right)\end{array}$ & AIC \\
\hline Putamen & $\begin{array}{c}0.292 \pm 0.053 \\
(1.7)\end{array}$ & $\begin{array}{c}0.073 \pm 0.022 \\
(15)\end{array}$ & $\begin{array}{c}0.133 \pm 0.030 \\
(21)\end{array}$ & $\begin{array}{c}0.043 \pm 0.007 \\
(8.6)\end{array}$ & $\begin{array}{c}4.25 \pm 1.07 \\
(13)\end{array}$ & $\begin{array}{c}3.19 \pm 0.97 \\
(17)\end{array}$ & $\begin{array}{c}17.3 \pm 4.6 \\
(2.0)\end{array}$ & $-43 \pm 21$ \\
\hline Caudate & $\begin{array}{c}0.248 \pm 0.047 \\
(1.7)\end{array}$ & $\begin{array}{c}0.051 \pm 0.022 \\
(19)\end{array}$ & $\begin{array}{c}0.110 \pm 0.063 \\
(38)\end{array}$ & $\begin{array}{c}0.051 \pm 0.014 \\
(17)\end{array}$ & $\begin{array}{c}5.71 \pm 2.69 \\
(18)\end{array}$ & $\begin{array}{c}2.09 \pm 0.93 \\
(27)\end{array}$ & $\begin{array}{l}16.2 \pm 5.5 \\
\quad(2.6)\end{array}$ & $-38 \pm 16$ \\
\hline Midbrain & $\begin{array}{c}0.203 \pm 0.044 \\
(2.6)\end{array}$ & $\begin{array}{c}0.095 \pm 0.026 \\
(13)\end{array}$ & $\begin{array}{c}0.053 \pm 0.028 \\
(34)\end{array}$ & $\begin{array}{c}0.042 \pm 0.009 \\
(22)\end{array}$ & $\begin{array}{c}2.18 \pm 0.37 \\
(11)\end{array}$ & $\begin{array}{c}1.29 \pm 0.55 \\
(18)\end{array}$ & $\begin{array}{c}4.9 \pm 1.1 \\
(3.8)\end{array}$ & $20 \pm 20$ \\
\hline Thalamus & $\begin{array}{c}0.269 \pm 0.042 \\
(1.7)\end{array}$ & $\begin{array}{c}0.123 \pm 0.024 \\
(6.0)\end{array}$ & $\begin{array}{c}0.029 \pm 0.018 \\
(26)\end{array}$ & $\begin{array}{c}0.041 \pm 0.023 \\
(23)\end{array}$ & $\begin{array}{c}2.26 \pm 0.55 \\
(4.7)\end{array}$ & $\begin{array}{c}0.71 \pm 0.20 \\
(12)\end{array}$ & $\begin{array}{c}3.8 \pm 0.8 \\
(3.8)\end{array}$ & $10 \pm 16$ \\
\hline Cerebellum & $\begin{array}{c}0.265 \pm 0.031 \\
(1.2)\end{array}$ & $\begin{array}{c}0.141 \pm 0.025 \\
(3.5)\end{array}$ & $\begin{array}{c}0.013 \pm 0.005 \\
(23)\end{array}$ & $\begin{array}{c}0.023 \pm 0.013 \\
(30)\end{array}$ & $\begin{array}{c}1.94 \pm 0.44 \\
(2.6)\end{array}$ & $\begin{array}{c}0.67 \pm 0.32 \\
(15)\end{array}$ & $\begin{array}{c}3.2 \pm 0.7 \\
(6.3)\end{array}$ & $-4 \pm 29$ \\
\hline
\end{tabular}

Values are mean \pm SD $(n=10)$, with percentage SE (which is inversely related to identifiability of parameters) in parentheses. 
TABLE 2

$B P_{\mathrm{ND}}$ Values by Indirect Kinetic and SRTM Methods

\begin{tabular}{lcc}
\hline & \multicolumn{2}{c}{$B P_{\mathrm{ND}}$} \\
\cline { 2 - 3 } Region & Indirect kinetic & SRTM \\
\hline Putamen & $4.46 \pm 0.95$ & $4.05 \pm 0.66$ \\
Caudate & $4.06 \pm 1.04$ & $3.61 \pm 0.67$ \\
Midbrain & $0.55 \pm 0.17$ & $0.62 \pm 0.13$ \\
Thalamus & $0.20 \pm 0.12$ & $0.29 \pm 0.08$
\end{tabular}

Values are mean $\pm \operatorname{SD}(n=10)$

approximately $15 \%-20 \%$ in the striatum and midbrain and approximately $30 \%$ in the thalamus, which were overall smaller than those by the indirect kinetic method. When $B P_{\mathrm{ND}}$ values estimated by the indirect kinetic method and by the SRTM method with 60-min data were compared with those estimated with 90 -min data, good correlations were observed ( $r=0.992$ for the indirect kinetic method and 0.999 for the SRTM method, $P<0.0001$ for both, Figs. $7 \mathrm{~A}$ and $7 \mathrm{~B})$.

\section{DISCUSSION}

${ }^{18} \mathrm{~F}-\mathrm{FE}-\mathrm{PE} 2 \mathrm{I}$ is a promising radioligand for quantifying DAT in healthy humans. The kinetics of ${ }^{18} \mathrm{~F}-\mathrm{FE}-\mathrm{PE} 2 \mathrm{I}$ were well described by a standard 2-TCM using the parent radioligand in plasma as the input function. Although the radiometabolites of ${ }^{18}$ F-FE-PE2I possibly have some effect on the radioactivity in the brain, the effect on the quantification was likely to be small. As a noninvasive quantification of DAT, the SRTM method was validated. The quantification was stable in both the striatum and the midbrain for both the indirect kinetic method with 2-TCM and the SRTM method with a scan duration of $60 \mathrm{~min}$.

General kinetics of ${ }^{18} \mathrm{~F}-\mathrm{FE}-\mathrm{PE} 2 \mathrm{I}$ showed promising characteristics, including a high specific-to-nonspecific ratio and relatively fast washout. Uptake was high in the putamen and caudate, relatively low in the midbrain and thalamus, and lowest in the cerebellum. In all target regions, specific binding reached maximum values within the duration of PET data acquisition, and transient equilibrium was reached during this acquisition period. Uptake in the midbrain was visualized as 2 distinct regions. Given the selectivity of ${ }^{18} \mathrm{~F}$-FE-PE2I for DAT in the midbrain shown by a displacement study (20), we suppose that this uptake reflected DAT binding, not serotonin transporter binding.

Compartment model analysis showed that the kinetics of ${ }^{18}$ F-FE-PE2I were well described by the 2-TCM using the parent radioligand in plasma as the input function. To estimate $B P_{\mathrm{ND}}$, we applied the indirect kinetic method instead of directly using the ratio of $k_{3} / k_{4}$ values. The $k_{3} / k_{4}$ ratio theoretically equals $B P_{\mathrm{ND}}$, but this estimate tends to be inaccurate because of data noise (29). We actually observed that these values showed poor identifiability and were not so reliably estimated (Table 1). Regional $B P_{\mathrm{ND}}$ values were

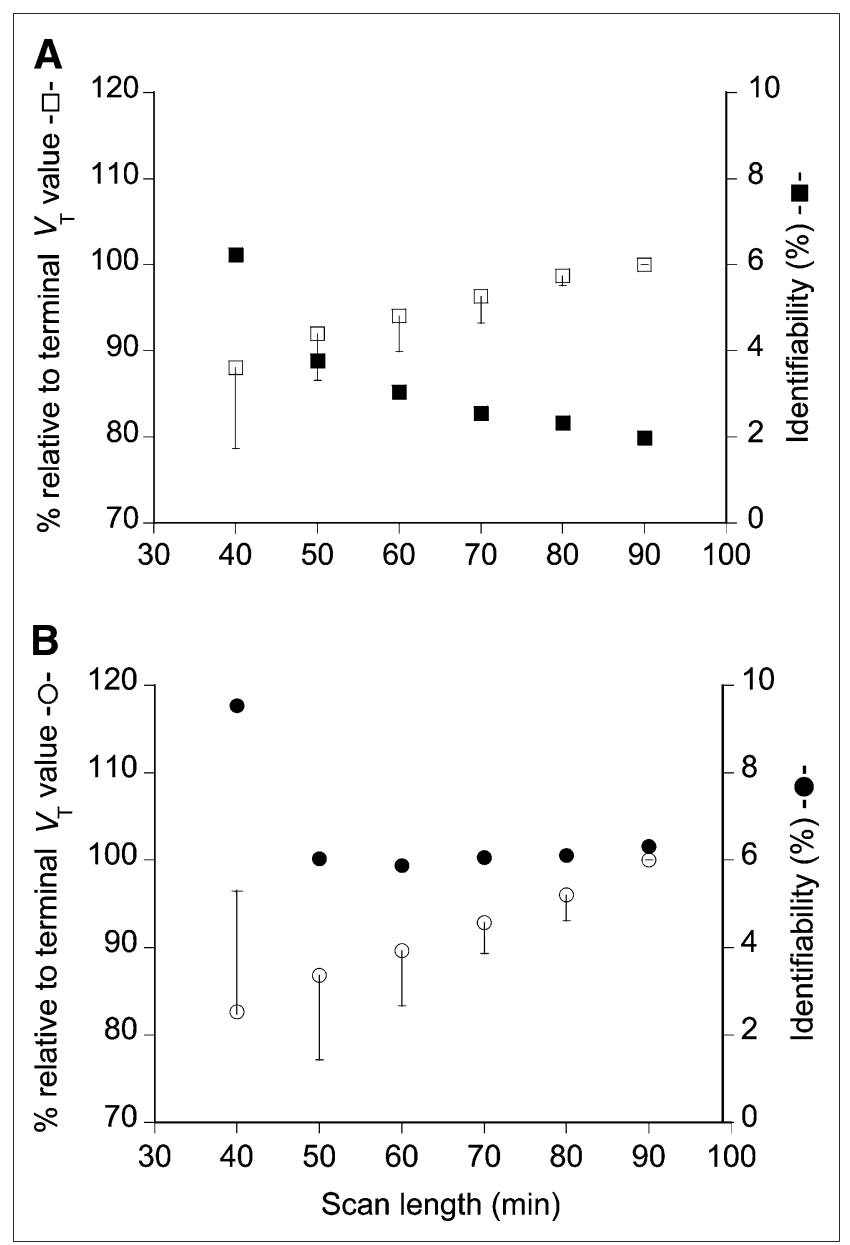

FIGURE 5. Value of $V_{\mathrm{T}}$ and identifiability as function of scan length. $V_{\mathrm{T}}$ and its corresponding SE (\%) (SE (\%)) were estimated in putamen (A) and cerebellum (B) with 2-TCM using parent as input function with truncating scan length from 90 to $40 \mathrm{~min}$. $V_{\mathrm{T}}$ values are expressed as percentage of terminal value and plotted along left $y$-axis for putamen $(\square)$ and cerebellum $(\bigcirc)$. Corresponding SE (\%), which is inversely related to identifiability, is plotted along right $y$-axis for putamen $(\square)$ and cerebellum $(\bullet)$. Error bar represents $\mathrm{SD}(n=10)$.

in accordance with previous autoradiographic (25) and in vivo studies $(16,17)$. As to intersubject variability, coefficients of variation of the $B P_{\mathrm{ND}}$ values were good in the putamen and caudate, acceptable in the midbrain, and poor in the thalamus. Because the $B P_{\mathrm{ND}}$ value in the midbrain was low ( $\sim 13 \%$ of that in the striatum) in comparison with the results of autoradiography $(\sim 50 \%$ of that in the striatum) (25), the $B P_{\mathrm{ND}}$ values in the midbrain could be affected by the partial-volume effect. Similar results were obtained in nonhuman primates using high-resolution research tomography. $B P_{\mathrm{ND}}$ in the midbrain was approximately $15 \%$ of the values in the striatum (21).

Although radiometabolites of ${ }^{18} \mathrm{~F}$-FE-PE2I possibly have some effects on radioactivity in the brain, their effect on quantification was likely to be small. HPLC analysis detected 2 radiometabolites of ${ }^{18} \mathrm{~F}-\mathrm{FE}-\mathrm{PE} 2 \mathrm{I}$ in plasma, 


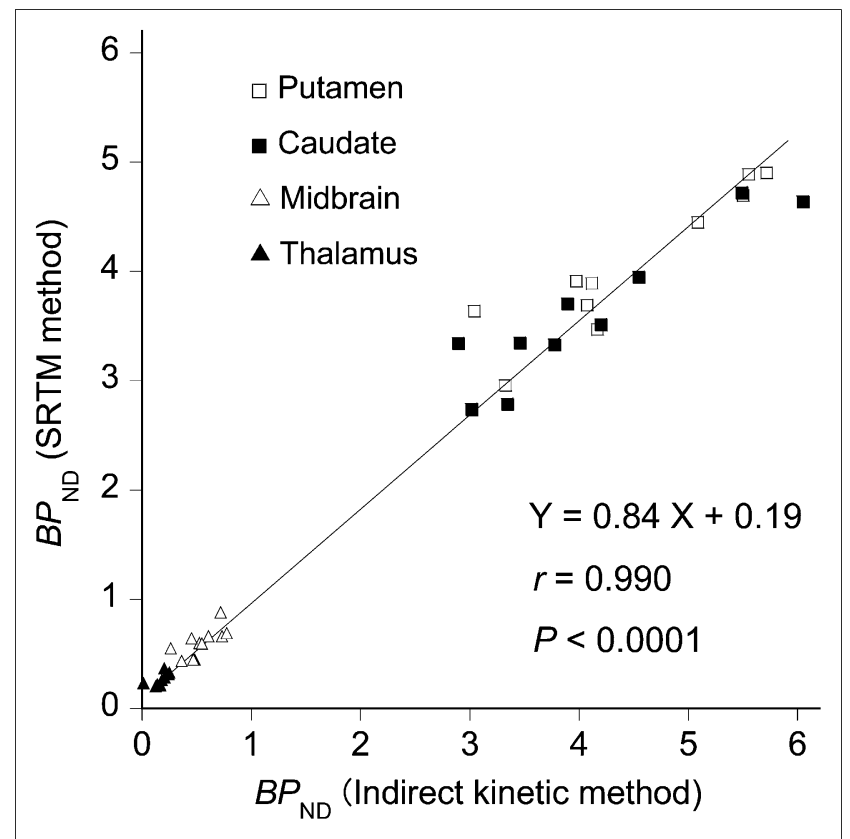

FIGURE 6. Correlation of $B P_{\mathrm{ND}}$ values estimated by indirect kinetic method using parent as input function and by SRTM method. $B P_{\mathrm{ND}}$ values showed significant correlation between 2 methods. Each data point represents $B P_{\mathrm{ND}}$ values in respective regions of each subject.

one with intermediate (M1) and the other with lower (M2) lipophilicity. M1 and M2 would be 4-hydroxymethyl and 4-carboxyl analogs of ${ }^{18} \mathrm{~F}-\mathrm{FE}-\mathrm{PE} 2 \mathrm{I}$, respectively, on the basis of the retention time of HPLC analysis, compared with the rat study (19). For clarity, in the following sentences, we refer to 4-hydroxymethyl and 4-carboxyl analogs of ${ }^{11} \mathrm{C}$-PE2I as $\mathrm{M} 1{ }^{\prime}$ and $\mathrm{M} 2$ ', respectively. In rats, M1' entered the brain, accumulated in the striatum to a lesser extent than ${ }^{11} \mathrm{C}$-PE2I, and metabolized to M2', which accumulated in the brain. Mainly as a result of the accumulation of $\mathrm{M}^{2}$, radioactivity in the cerebellum of rats did not decrease from $55 \mathrm{~min}$ to the end of the scan (120 min) (19). Whereas in the current study with ${ }^{18}$ F-FE-PE2I, radioactivity in the cerebellum showed a gradual decrease to the end of the scan (90 min) (Fig. 2A), indicating that the amount of retention of radiometabolites in the brain in humans with ${ }^{18} \mathrm{~F}$-FE-PE2I was less than that in rats with ${ }^{11} \mathrm{C}$-PE2I. This result was in accordance with the study in monkeys with ${ }^{18}$ F-FE-PE2I $(21)$.

The effects of radiometabolites on the quantification were small in the target and reference regions. To evaluate the effect of the possible accumulation of radiometabolites on the estimation of $V_{\mathrm{T}}$, we performed a time-stability analysis of $V_{\mathrm{T}}$. The increase in $V_{\mathrm{T}}$ with an increase in scan length could be interpreted as evidence for the accumulation of radiometabolites in the brain (30). In the current study, $V_{\mathrm{T}}$ values by the 2-TCM in the putamen and cerebellum with 60-min data were approximately $94 \%$ and $90 \%$ of terminal values with 90-min data (Fig. 6). This finding
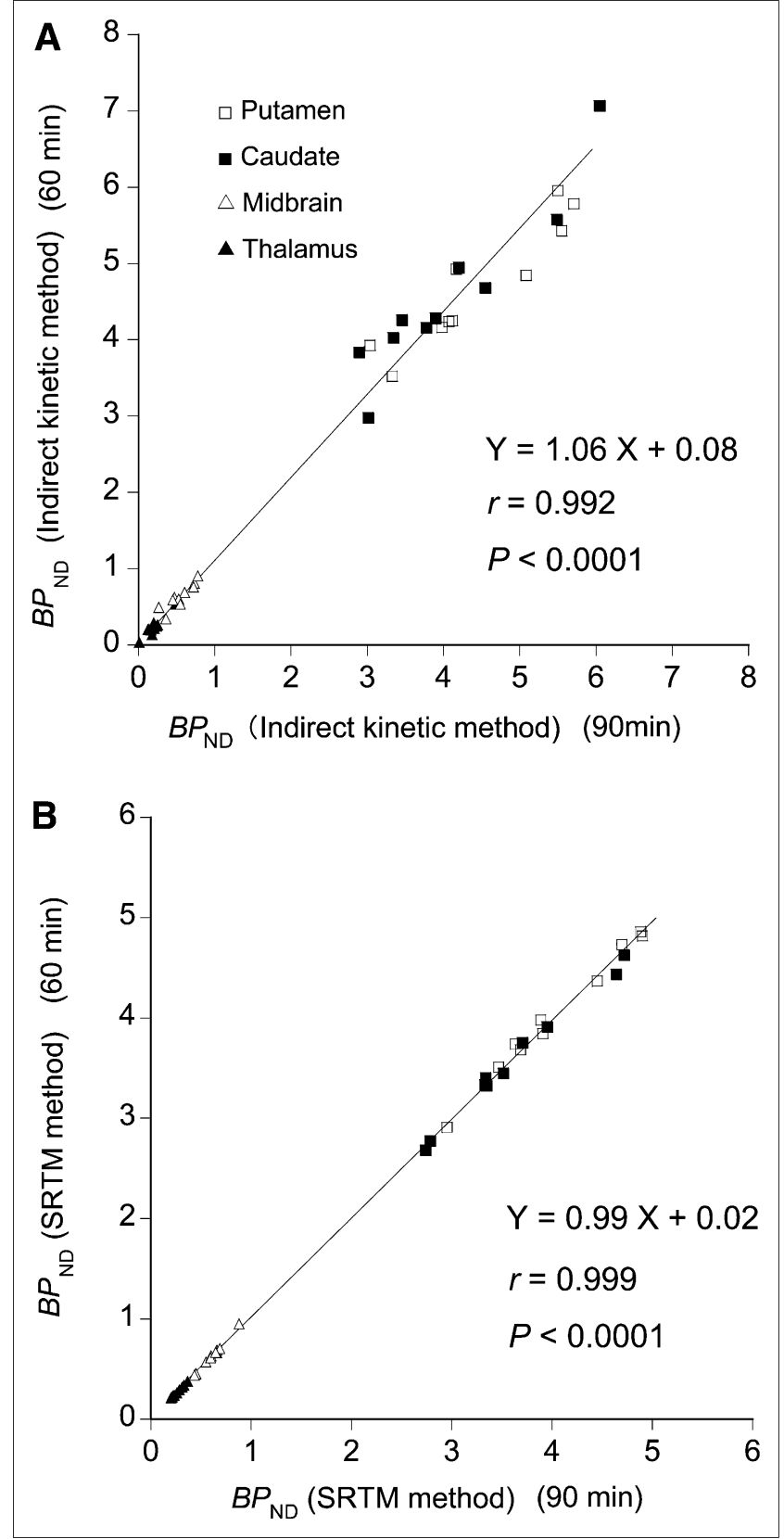

FIGURE 7. Correlation of $B P_{\mathrm{ND}}$ values estimated with 60 - and 90-min data. (A) $B P_{\mathrm{ND}}$ values estimated by indirect kinetic method. (B) $B P_{\mathrm{ND}}$ values estimated by SRTM method. Significant correlations were observed in both methods between estimates with 60- and 90-min data.

might indicate that $V_{\mathrm{T}}$ values were slightly affected by the radiometabolites, but the effects were relatively small.

To estimate the possible bias by the radiometabolites on $V_{\mathrm{T}}$, we tried a combined input function of the parent and the radiometabolite M1 as previously performed by Varrone et al. in nonhuman primates using ${ }^{18}$ F-FE-PE2I (21). The main assumption of this approach is that the parent and the radiometabolite M1 would behave similarly and hence could be combined in a single input. With this approach, 
the 2-TCM yielded approximately 30\%-35\% lower estimates of $V_{\mathrm{T}}$ values in the striatum and midbrain than with the 2-TCM using the parent as the input function. The decrease of $V_{\mathrm{T}}$ could be a possible maximum bias by the radiometabolites. However, the $V_{\mathrm{T}}$ values estimated by the 2-TCM using a summation of the parent and M1 as the input function were not well identified in some subjects in the thalamus and in most in the cerebellum. Because combining the parent and M1 as the input function did not improve the fitting, at least for these regions the assumption was not fully supported by the data. Identification of the radiometabolites of ${ }^{18}$ F-FE-PE2I and assessment of their affinity for the DAT would be necessary to confirm or rule out the above assumption.

As a less noninvasive quantification of DAT without arterial blood data, $B P_{\mathrm{ND}}$ values were well estimated by the SRTM method using the cerebellum as the reference region. $B P_{\mathrm{ND}}$ values estimated by the SRTM method were well correlated with those estimated by the indirect kinetic method with the 2-TCM, which was not so much affected by radiometabolites as we discussed above. The SRTM method yielded slight underestimation and overestimation of $B P_{\mathrm{ND}}$ values in regions with high and low DAT densities, respectively, although the magnitude of the bias was small ( $\sim 10 \%$ in the putamen, caudate, and midbrain). This bias seems to be an intrinsic limitation of the SRTM method because a similar phenomenon was observed in other ligands, including the serotonin 5-hydroxytryptamine-1A ligand ${ }^{11} \mathrm{C}$-WAY-100653 (31), perhaps because of a violation of the assumptions of SRTM method. The intersubject variability of $B P_{\mathrm{ND}}$ values by the SRTM method were better than those by the indirect kinetic method with the 2-TCM, indicating that the SRTM method provided more precise estimation of $B P_{\mathrm{ND}}$ in all regions, including the midbrain.

Time-stability analysis indicated that 60-min scan duration is enough to estimate DAT binding with ${ }^{18} \mathrm{~F}-\mathrm{FE}-\mathrm{PE} 2 \mathrm{I}$ in humans. A recent study in nonhuman primates indicated that an approximately 60-min scan length was sufficient for the quantification of DAT with ${ }^{18} \mathrm{~F}-\mathrm{FE}-\mathrm{PE} 2 \mathrm{I}$ by the SRTM method (32). In the current study in humans, quantification was stable for both the indirect kinetic method and SRTM method with a scan duration of $60 \mathrm{~min}$. Good correlations were observed between $B P_{\mathrm{ND}}$ values estimated with 60- and 90-min data for both the indirect kinetic method and the SRTM method.

${ }^{18}$ F-FE-PE2I has many desirable characteristics among DAT ligands available for human imaging. High affinity and high selectivity for DAT allowed reliable quantification of specific binding not only in the striatum but also in the midbrain. Because of the faster kinetics even in highdensity regions (i.e., the striatum), quantification with a shorter time (60 min) was possible, and the SRTM method yielded less biased $B P_{\mathrm{ND}}$ in the striatum than ${ }^{11} \mathrm{C}-\mathrm{PE} 2 \mathrm{I}$. In addition, labeling with ${ }^{18} \mathrm{~F}$, which has a longer half-life than ${ }^{11} \mathrm{C}$ (110 vs. $20 \mathrm{~min}$ ), allows distribution of the radioligand to PET centers without a cyclotron.
${ }^{18} \mathrm{~F}$-labeled DAT ligands previously reported in humans include ${ }^{18} \mathrm{~F}$-FPCIT (12), ${ }^{18} \mathrm{~F}$-CFT $\left({ }^{18} \mathrm{~F}\right.$-WIN35,428) (11), and ${ }^{18}$ F-FECNT (13). All of these ligands have a high affinity for DAT, and the maximum striatum-to-cerebellum ratios have been reported to be approximately 3.5-9.0. The possible advantage of ${ }^{18} \mathrm{~F}$-FPCIT and ${ }^{18} \mathrm{~F}$-FECNT could be the absence of potentially BBB-permeable radiometabolites. However, the disadvantage of those ligands was that the kinetics are slow, requiring over 90 min to reach peak brain uptake in the striatum. Among ${ }^{18} \mathrm{~F}$-labeled DAT ligands, ${ }^{18}$ F-FE-PE2I has a relatively high striatum-tocerebellum ratio $(\sim 7.0)$ and obviously the fastest kinetics.

One of the 2 limitations of the current study is the absence of the measurement of the free fraction in plasma, which would enable us to measure DAT density more accurately. Another limitation is the absence of an animal ex vivo study using ${ }^{18} \mathrm{~F}-\mathrm{FE}-\mathrm{PE} 2 \mathrm{I}$ to examine the BBB permeability and affinity for DAT of the radiometabolites. These issues should be explored in future studies.

\section{CONCLUSION}

${ }^{18} \mathrm{~F}-\mathrm{FE}-\mathrm{PE} 2 \mathrm{I}$ is a promising radioligand for quantifying DAT in healthy humans. The kinetics of ${ }^{18} \mathrm{~F}-\mathrm{FE}-\mathrm{PE} 2 \mathrm{I}$ were well described by a standard 2-TCM using the parent radioligand in plasma as the input function. Although radiometabolites of ${ }^{18}$ F-FE-PE2I possibly have some effect on the radioactivity in the brain, their effect on quantification was likely to be small. As a noninvasive quantification of DAT, the SRTM method was validated. The quantification was stable in both the striatum and the midbrain for both the indirect kinetic method with 2-TCM and the SRTM method with a scan duration of $60 \mathrm{~min}$, although the SRTM method yielded a slight underestimation and overestimation of $B P_{\mathrm{ND}}$ values in regions with high and low DAT densities, respectively. If no major differences in metabolism between patients and controls are present in clinical studies, noninvasive estimation of $B P_{\mathrm{ND}}$ by the SRTM method with a 60-min scan will be sufficiently accurate for DAT quantification.

\section{DISCLOSURE STATEMENT}

The costs of publication of this article were defrayed in part by the payment of page charges. Therefore, and solely to indicate this fact, this article is hereby marked "advertisement" in accordance with 18 USC section 1734.

\section{ACKNOWLEDGMENTS}

We thank Katsuyuki Tanimoto, Takahiro Shiraishi, and Takehito Ito for their assistance in performing PET experiments and Izumi Izumida and Kazuko Suzuki for their help as clinical research coordinators. This study was supported by a grant-in-aid for Molecular Imaging Program from the Ministry of Education, Culture, Sports, Science and Technology and by a Health Labour Sciences Research 
grant from the Ministry of Health, Labour and Welfare, Japanese government. No other potential conflict of interest relevant to this article was reported.

\section{REFERENCES}

1. Hurley MJ, Mash DC, Jenner P. Markers for dopaminergic neurotransmission in the cerebellum in normal individuals and patients with Parkinson's disease examined by RT-PCR. Eur J Neurosci. 2003;18:2668-2672.

2. Ginovart N, Lundin A, Farde L, et al. PET study of the pre- and post-synaptic dopaminergic markers for the neurodegenerative process in Huntington's disease. Brain. 1997;120:503-514

3. Jucaite A, Fernell E, Halldin C, Forssberg H, Farde L. Reduced midbrain dopamine transporter binding in male adolescents with attention-deficit/hyperactivity disorder: association between striatal dopamine markers and motor hyperactivity. Biol Psychiatry. 2005;57:229-238.

4. Nakamura K, Sekine Y, Ouchi Y, et al. Brain serotonin and dopamine transporter bindings in adults with high-functioning autism. Arch Gen Psychiatry. 2010;67:59-68.

5. Arakawa R, Ichimiya $\mathrm{T}$, Ito $\mathrm{H}$, et al. Increase in thalamic binding of $\left[{ }^{11} \mathrm{C}\right] \mathrm{PE} 2 \mathrm{I}$ in patients with schizophrenia: a positron emission tomography study of dopamine transporter. J Psychiatr Res. 2009;43:1219-1223.

6. Fowler JS, Volkow ND, Wolf AP, et al. Mapping cocaine binding sites in human and baboon brain in vivo. Synapse. 1989;4:371-377.

7. Wong DF, Yung B, Dannals RF, et al. In vivo imaging of baboon and human dopamine transporters by positron emission tomography using $\left[{ }^{11} \mathrm{C}\right]$ WIN 35,428 . Synapse. 1993;15:130-142.

8. Farde L, Halldin C, Muller L, Suhara T, Karlsson P, Hall H. PET study of $\left[{ }^{11} \mathrm{C}\right]$ beta-CIT binding to monoamine transporters in the monkey and human brain. Synapse. 1994;16:93-103.

9. Ding YS, Fowler JS, Volkow ND, et al. Pharmacokinetics and in vivo specificity of $\left[{ }^{11} \mathrm{C}\right] \mathrm{dl}$-threo-methylphenidate for the presynaptic dopaminergic neuron. Synapse. 1994;18:152-160.

10. Fischman AJ, Bonab AA, Babich JW, et al. $\left[{ }^{11} \mathrm{C},{ }^{127} \mathrm{I}\right]$ Altropane: a highly selective ligand for PET imaging of dopamine transporter sites. Synapse. 2001;39:332-342.

11. Laakso A, Bergman J, Haaparanta M, Vilkman H, Solin O, Hietala J. $\left[{ }^{18} \mathrm{~F}\right] \mathrm{CFT}$ ( $\left[{ }^{18}\right.$ F $]$ WIN 35,428], a radioligand to study the dopamine transporter with PET: characterization in human subjects. Synapse. 1998;28:244-250.

12. Kazumata K, Dhawan V, Chaly T, et al. Dopamine transporter imaging with fluorine-18-FPCIT and PET. J Nucl Med. 1998;39:1521-1530.

13. Davis MR, Votaw JR, Bremner JD, et al. Initial human PET imaging studies with the dopamine transporter ligand ${ }^{18}$ F-FECNT. J Nucl Med. 2003;44:855-861.

14. Emond P, Garreau L, Chalon S, et al. Synthesis and ligand binding of nortropane derivatives: N-substituted 2beta-carbomethoxy-3beta-(4'-iodophenyl)nortropane and N-(3-iodoprop-(2E)-enyl)-2beta-carbomethoxy-3beta-( $3^{\prime}, 4^{\prime}$-disubstituted phenyl)nortropane: new high-affinity and selective compounds for the dopamine transporter. J Med Chem. 1997;40:1366-1372.

15. Halldin C, Erixon-Lindroth N, Pauli S, et al. $\left[{ }^{11} \mathrm{C}\right] \mathrm{PE} 2 \mathrm{I}$ : a highly selective radioligand for PET examination of the dopamine transporter in monkey and human brain. Eur J Nucl Med Mol Imaging. 2003;30:1220-1230.
16. Jucaite A, Odano I, Olsson H, Pauli S, Halldin C, Farde L. Quantitative analyses of regional $\left[{ }^{11} \mathrm{C}\right] \mathrm{PE} 2 \mathrm{I}$ binding to the dopamine transporter in the human brain: a PET study. Eur J Nucl Med Mol Imaging. 2006;33:657-668.

17. Hirvonen J, Johansson J, Teras M, et al. Measurement of striatal and extrastriatal dopamine transporter binding with high-resolution PET and $\left[{ }^{11} \mathrm{C}\right] \mathrm{PE} 2 \mathrm{I}$ : quantitative modeling and test-retest reproducibility. J Cereb Blood Flow Metab. 2008;28:1059-1069.

18. Seki C, Ito H, Ichimiya T, et al. Quantitative analysis of dopamine transporters in human brain using $\left[{ }^{11} \mathrm{C}\right] \mathrm{PE} 2 \mathrm{I}$ and positron emission tomography: evaluation of reference tissue models. Ann Nucl Med. 2010;24:249-260.

19. Shetty HU, Zoghbi SS, Liow JS, et al. Identification and regional distribution in rat brain of radiometabolites of the dopamine transporter PET radioligand $\left[{ }^{11} \mathrm{C}\right]$ PE2I. Eur J Nucl Med Mol Imaging. 2007;34:667-678.

20. Varrone A, Steiger C, Schou M, et al. In vitro autoradiography and in vivo evaluation in cynomolgus monkey of $\left[{ }^{18} \mathrm{~F}\right] \mathrm{FE}-\mathrm{PE} 2 \mathrm{I}$, a new dopamine transporter PET radioligand. Synapse. 2009;63:871-880.

21. Varrone A, Toth M, Steiger C, et al. Kinetic analysis and quantification of the dopamine transporter in the nonhuman primate brain with ${ }^{11} \mathrm{C}$-PE2I and ${ }^{18} \mathrm{~F}$-FEPE2I. J Nucl Med. 2011;52:132-139.

22. Schou M, Steiger C, Varrone A, Guilloteau D, Halldin C. Synthesis, radiolabeling and preliminary in vivo evaluation of $\left[{ }^{18} \mathrm{~F}\right] \mathrm{FE}-\mathrm{PE} 2 \mathrm{I}$, a new probe for the dopamine transporter. Bioorg Med Chem Lett. 2009;19:4843-4845.

23. Innis RB, Cunningham VJ, Delforge J, et al. Consensus nomenclature for in vivo imaging of reversibly binding radioligands. J Cereb Blood Flow Metab. 2007;27: 1533-1539.

24. Leenders KL, Perani D, Lammertsma AA, et al. Cerebral blood flow, blood volume and oxygen utilization: normal values and effect of age. Brain. 1990; 113:27-47.

25. Hall H, Halldin C, Guilloteau D, et al. Visualization of the dopamine transporter in the human brain postmortem with the new selective ligand [ ${ }^{125}$ I]PE2I. Neuroimage. 1999;9:108-116.

26. Lammertsma AA, Hume SP. Simplified reference tissue model for PET receptor studies. Neuroimage. 1996;4:153-158.

27. Akaike H. A new look at the statistical model identification. IEEE Trans Automat Contr. 1974;19:716-723.

28. Carson R. Parameters estimation in positron emission tomography. In: Phelps M, Mazziotta J, Schelbert H, eds. Positron Emission Tomography Principle Applications for the Brain and the Heart. New York, NY: Raven Press; 1986:347-390.

29. Seneca N, Skinbjerg M, Zoghbi SS, et al. Kinetic brain analysis and whole-body imaging in monkey of $\left[{ }^{11} \mathrm{C}\right] \mathrm{MNPA}$ : a dopamine agonist radioligand. Synapse. 2008;62:700-709.

30. Terry G, Liow JS, Chernet E, et al. Positron emission tomography imaging using an inverse agonist radioligand to assess cannabinoid CB1 receptors in rodents. Neuroimage. 2008;41:690-698.

31. Parsey RV, Slifstein M, Hwang DR, et al. Validation and reproducibility of measurement of 5-HT1A receptor parameters with [carbonyl- ${ }^{11} \mathrm{C}$ ]WAY-100635 in humans: comparison of arterial and reference tisssue input functions. $J$ Cereb Blood Flow Metab. 2000;20:1111-1133.

32. Varrone A, Gulyas B, Takano A, Stabin MG, Jonsson C, Halldin C. Simplified quantification and whole-body distribution of $\left[{ }^{18} \mathrm{~F}\right]$ FE-PE2I in nonhuman primates: prediction for human studies. Nucl Med Biol. 2012;39:295-303. 